\title{
Regulatory Framework for Academic Investigator-Sponsored Investigational New Drug Development of Cell and Gene Therapies in the USA
}

\author{
Anindya Dasgupta ${ }^{1}$ (D) Kristen Herzegh $^{2} \cdot$ H. Trent Spencer ${ }^{3} \cdot$ Christopher Doering $^{3} \cdot$ Eric Day $^{1} \cdot$ William P. Swaney $^{1}$
}

Accepted: 27 July 2021 / Published online: 30 September 2021

(c) The Author(s), under exclusive licence to Springer Nature Switzerland AG 2021

\begin{abstract}
Purpose of Review The promise of cell and gene therapy (CGT) products for a multitude of diseases has revitalized investigators to advance novel CGT product candidates to first-in-human trials by pursuing the investigational new drug (IND) mechanism administered by the United States (US) Food and Drug Administration (FDA). This review is intended to familiarize academic investigators with the IND governing regulations set forth by the FDA.

Recent Findings CGT products are extraordinarily complex biologics and, therefore, early-stage evaluation programs must be customized to satisfactorily address their unique developmental challenges. The US FDA continues to foster the development of transformational technology that will facilitate the broad application of safe and effective gene therapy products that have the potential to alleviate many conditions previously out of reach of therapeutic intervention. FDA is committed to working with the scientific community and industry to facilitate the availability of these treatments to patients.

Summary The pathway to meet regulatory compliance during early stage IND programs can be daunting to academic investigators interested in CGT product development that typically don't progress beyond phase $1 / 2$. However, by keeping abreast of current regulatory framework and building upon FDA's supportive infrastructure, an investigator can be well-positioned to advance innovative scientific discoveries towards early stage clinical assessments.
\end{abstract}

Keywords FDA $\cdot$ Regulatory $\cdot$ IND $\cdot$ Cell and gene therapy

\section{Introduction}

Under US federal law, chemical or biological articles that are intended to diagnose, cure, treat, mitigate, or prevent disease, or are intended to affect the structure or function of the body, are defined as drugs. Biologics, such as cell

This article is part of the Topical Collection on Policy: From Clinic to Bench

Anindya Dasgupta

adasgupta@expressiontherapeutics.com

1 Expression Manufacturing LLC, West Chester, OH, USA

2 Marcus Center for Pediatric Cellular Therapies, Aflac Cancer and Blood Disorders Center, Children's Healthcare of Atlanta and Emory University, Atlanta, GA, USA

3 Cell and Gene Therapy Program, Department of Pediatrics, Aflac Cancer and Blood Disorders Center, Children's Healthcare of Atlanta and Emory University, Atlanta, GA, USA and gene therapy (CGT) products that share such attributes as described above are therefore drugs. Drugs and biological products intended for use in humans in the USA are regulated by the Food and Drug Administration (FDA), an agency under the US Department of Health and Human Services (HHS). A set of federal laws and codifications of regulations, Code of Federal Regulations (CFR), as described under the Federal Food, Drug, and Cosmetic Act (FD\&C Act) and the Public Health Service Act (PHS Act) bestow such authority to FDA [1]. The FDA is composed of nine center level organizations. Of these, the Center for Drug Evaluation and Research (CDER), Center for Devices and Radiological Health (CDRH), and the Center for Biologics Evaluation and Research (CBER) are primarily tasked with regulatory oversight of clinical trials involving investigational drugs and biological products. Each center has several offices to oversee various aspects of the regulatory framework. CBER's mission is to protect and enhance the public health through the regulation of biological products including blood, vaccines, allergenics, human cells or tissue, 
and CGTs. The office of Tissues and Advanced Therapies (OTAT) within CBER is currently charged to oversee cellular therapies.

CGT products encompass cellular products that include stem cells and their derivatives, cellular immunotherapeutic, and gene therapy products that include viral and nonviral-based vectors and ex vivo manipulated bioengineered immune cells. The field of CGT products has overcome early periods of great uncertainty which saw serious adverse events leading to skepticism towards the durability of such treatments and has now entered an era of exciting and promising growth [2-5]. It must be noted that clinical trials with experimental treatment modalities, including CGT-based approaches, can lead to unforeseen adverse events and deaths which must be promptly reported to the FDA using established reporting mechanisms. These reports may also be disseminated to key stakeholders via presentation at prominent meetings, publications in scientific and media outlets to inform clinicians and the interested public on the current state on the usage of such therapeutic interventions [6, 7]. In 2019, the majority of CGT-based IND submissions were geared towards the development of therapies against hematological and solid cancers while the investigational agents to combat infectious diseases, autoimmune disorders and other diseases constitute the remaining pool of applications (Fig. 1) [8]. Both technological advancement and growing experience in conducting clinical trials have contributed to an increase in benefit to risk ratio of such therapeutics. Such refinements have drawn renewed interest within the biotechnological companies to capitalize on this market alongside traditional academic investigators

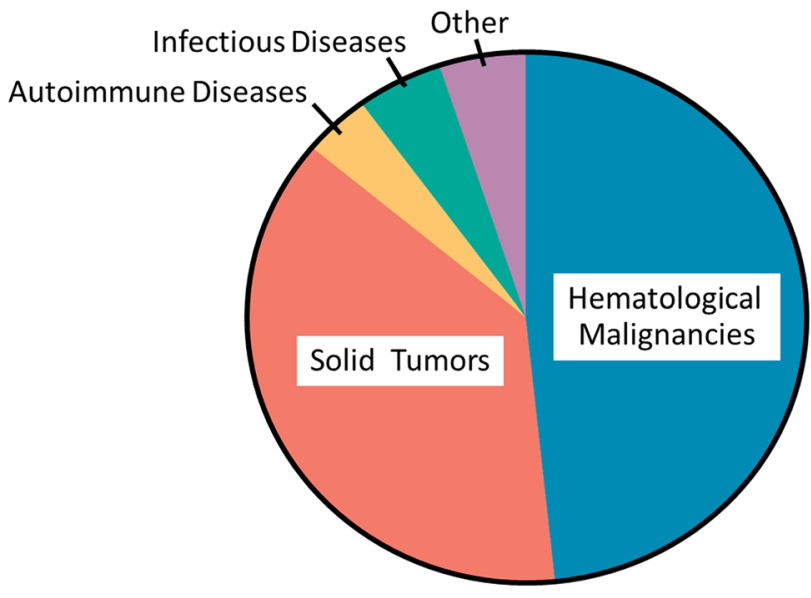

Fig. 1 Distribution of product indications for all cell and gene immunotherapy IND submissions to the FDA in 2019. Reproduced with permission: Copyright (C) 2020 Galaro AK \& Saeui C. Published by Cell and Gene Therapy Insights under Creative Commons License Deed CC BY NC ND 4.0: https://www.insights.bio/cell-and-gene-therapy-insights/ journal/article/1787/FDA-perspective-on-the-preclinical-developmentof-cell-based-immunotherapies restricted primarily to early stage developments. Consequently, the number of IND submissions have risen exponentially over the last 5 years with more than 900 applications last year (2020) alone compared to about 150 submissions in the year earlier [9]. Keeping up with such a meteoric growth is FDA's prediction that from year 2025 onwards the agency may approve between 10 to 20 CGT based therapeutics every year [10•]. Investigators in academic centers will continue to be valuable players in this space which benefited from the early-stage developments of a majority of approved products with genetic modifications that were initiated in academia [9]. Currently, there are nineteen FDA-approved cellular and gene therapy products of which the included eight blood products are regulated in a different manner than the majority of the CGT products. [11]. With the intent to benefit patients with significant unmet clinical needs comes the risk of introducing potential serious adverse events. Such effects may include the potential for inappropriate cell proliferation, risks of insertional mutagenesis and onset of cytokine storm after adoptive transfer, all of which continue to direct drug development programs. Towards this end, the FDA's modern regulatory framework supports risk mitigation strategies to increase efficiency in the much-needed exploration of these therapy approaches.

Stakeholders engaged in developing CGT-based drug products that are intended to be evaluated for investigational use only are exempted under FD\&C Act 505(i) from filing a New Drug Application (NDA). The route to apply for such exemption is called an Investigational New Drug Application (IND) which is codified in Title 21 of the Code of Federal Regulations, Part 312 (21 CFR 312). It is assumed that such investigational drugs would require interstate shipping for evaluation and testing purposes. The exemption from the federal law, 21 CFR 312.1, that authorizes movement for drugs requiring an approved marketing application such as NDA or Biologics License Application (BLA), allows for such free movement of the investigational drugs across the country [1]. A distinguishing feature of the IND is that it is neither approved nor disapproved. The sponsor applies for an IND designation, receives a receipt from FDA acknowledging the filing and waits for a mandatory period of 30 days from the receipt of such a letter before commencing the clinical trial. However, the FDA has the authority to ask for clarifications, place a partial or a full hold on the proposed clinical trial under 21 CFR 312.42. The sponsor must produce enough evidence to clarify any concern from FDA before proceeding with the trial. If a partial hold is in place, then the sponsor can start the trial with the components not under hold. However, no portion of the trial can be initiated if the full hold is in place and the trial can commence only after the underlying cause of such an action is resolved [12]. Besides the FDA, the investigator or sponsor must also adhere to procedures set forth by the host institute to enable 
studies with biological materials. Such policies are enacted upon the guidelines provided by the federal authority such as National Institute of Health (NIH) and regulations on public health and environment protection from state and local communities. The key governing bodies that are charged to enforce such directives are listed in Table 1.

The primary objective of the FDA is patient safety while creating a supportive and encouraging environment by affording freedom, at the early stages of innovative drug development, to the investigator for selecting proper study designs and rationale for a clinical trial. Towards this end, the FDA issues guidance documents at appropriate intervals $[13,14]$. At any time the FDA sees appropriate, such as to clarify and/or update an existing document, it publishes draft guidance documents in the Federal Register which is governed by the National Archives and Records Administration, and provides a time period for comments from the stakeholders prior to the issuance of the final document. After taking into accounts of such responses, the FDA publishes the "final guidance document." Stakeholders should adhere to the recommendations as set forth in these documents. However, considering the unique diversity and biology of the CGT products, tailor made developmental pathways are often crafted in consultations with the FDA. The latest guidance document to impact CGT-based product developments acknowledges that the materials for pre-clinical testing may differ from the final therapeutic products for early-stage clinical trials. In such circumstances, it is recommended that an early stage IND submission describes such differences with regard to product safety, especially for their proposed use in first in human $(\mathrm{FIH})$ trials and activity [15••]. The guidances are nonbinding and legally nonenforceable recommendations unless specific regulatory or statutory requirements are cited. The FDA relies upon the user-dependent understanding and interpretations to align them to its current thinking. If the FDA feels that such user-reliance leads to occasions where the user is not taking full advantage afforded by an existing guidance, then a new guidance document is issued.

An IND can originate from (i) commercial enterprise such as pharmaceutical/biotechnological/governmental/nonacademic entities or (ii) an individual academic investigator. In contrast to a commercial venture that typically has access to resource and logistics for preparation of an IND document and partake multi-site clinical trial, an academic investigator can find it challenging to navigate the complex network of regulatory requirements towards an IND submission and to conduct single site trials. The FDA considers phase 2 and 3 submissions commercial. Investigators may submit a waiver should the sole focus of the studies be for research purposes only. This review describes the IND mechanism as it relates to such an investigator engaged primarily in earlystage evaluations of investigational CGT products.

\section{Roadmap for an Early-Stage CGT Product-Based IND Program}

The IND mechanism provides a springboard for investigators to carry their preliminary evaluations to advanced clinical stages. The critical attributes to navigate an earlystage IND pathway are as follows: (i) identification of the drug candidate and its evaluation in a nonclinical setting, (ii) implementation of scientific and regulatory strategies to transition the potential therapeutic benefits derived from pre-clinical animal models towards FIH trial as required for IND filing, and (iii) conduct the clinical trial pursuant to the regulatory oversights of the host institution and the FDA. Key steps that are typically encountered during such a journey are described below.

Table 1 Examples of host institution research oversite

\begin{tabular}{ll}
\hline Host institutions research oversite (examples) & Oversite responsibilities \\
\hline Institutional Review Board (IRB) & Tasked to review any investigation comprising of human subjects \\
$\begin{array}{l}\text { Data Safety and Monitoring Board/Committee } \\
\text { (DSMB/C) }\end{array}$ & $\begin{array}{c}\text { Ensure that study protocols provide provisions to ensure the safety of trial participants } \\
\text { and monitors all aspects related to data integrity such as its accuracy and validation } \\
\text { Institutional Biosafety Committee (IBC) }\end{array}$ \\
$\begin{array}{l}\text { Entrusted to oversee all safety assessments and contingency plans to manage exposure } \\
\text { to engineered genetic material and other biohazardous agents }\end{array}$ \\
$\begin{array}{l}\text { Management and dispensing of investigational drugs } \\
\text { Research Health and Safety Committee (RHSC) }\end{array}$ & $\begin{array}{l}\text { Review research conducted with, but not limited to, biological toxins, samples of } \\
\text { human origin including human cell lines, tissues, arthropods, nanoparticles, and } \\
\text { microorganisms }\end{array}$ \\
Institutional Animal Care and Use Committee IACUC & Oversite of animal care (applicable to pre-clinical IND studies)
\end{tabular}

CGT-based IND studies are typically approved by the IRB pursuant to a satisfactory review by both IBC and DSMB/C as well as the receipt of a "Study may Proceed" letter from the FDA upon request from the sponsor 


\section{Exploratory Studies to Evaluate the Suitability of the Investigational Drug for IND Designation}

As described below, an investigational drug development scheme is initiated by undertaking preliminary evaluation which intensifies to studies suitable for IND filling, assuming the product shows therapeutic benefit and is deemed potentially safe for human trial.

Discovery Phase with Pre-clinical POC Studies. Basic research on a conceptual idea takes shape during this time. In this phase, initial identification and evaluation of the innovative drug candidate and its disease modifying action is evaluated. Towards this end, decisions are drawn on existing literature or new ideas on the selection of several components such as (i) adoptive cell product, either naïve or gene-modified, (ii) cell source such as established or primary cell lines with the desired properties or discovery/development of a new cell line, (iii) viral vectors for either ex-vivo cell manipulation or in vivo vector delivery, and (iv) potential phase compliant GMP manufacturing procedures. The proof-of-concept (POC) studies, in the initial stage, typically involve both in vitro and in vivo animal (nonclinical) studies.

Outcomes from animal model-based evaluations of the investigational product are important determinants for its potential progression to a clinical trial. Therefore, the selection criteria of an animal model are crucial and are based on various factors such as (i) recapitulation and pathophysiology of the targeted disorder and (ii) permissiveness to the therapeutic modality [16]. The investigator must be aware of limitations posed by the employment of in vivo models in their abilities to closely mimic human response to the administered test product. Immunocompromised hosts, such as NOD SCID mice are often employed when the product candidate is a human cellbased therapy. However, their use is limited, and evaluation of drug-induced immune responses is not possible, as these mice are immunocompromised. During the designing of animal studies, the investigator is encouraged, in principle, to (i) follow the recommendation from National Toxicology Program Interagency Center for the Evaluation of Alternative Toxicological Methods (NICEATM) to reduce animal use by adhering to the three ' $R$ ' principles of replace, reduce, or refine their usage without compromising on safety and toxicity data [17], (ii) plan for study durations with scheduled evaluations at several time points to adequately capture biological response, (iii) incorporate sufficient number of animals to derive targeted biological response with statistically meaningful outcomes, and (iv) incorporated a strategy of blinded studies where the research staff do not know the controls from the drug product candidate.
Pre-clinical IND Enabling Studies. The main objective of such studies is to evaluate if the investigational product has the appropriate risk to benefit profile for the intended indication. Towards this end intensification and refinements to the pre-clinical studies are designed to include pharmacological and toxicological profile of the investigational drug along with its efficacy, biodistribution, starting dose, and dosing regimen as described later [17]. Evaluation of pre-clinical toxicological studies are typically required to be performed at a testing facility compliant to Good Laboratory Practice (GLP) as per 21 CFR part 58 [18, 19]. However, toxicological studies with CGT-derived products frequently require (i) unique on-site animal care, and (ii) in-house expertise for end point analysis from animals exposed to bioengineered products. Such studies can be undertaken non-GLP provided those aspects of GLP not performed are identified and the studies performed in-house in facilities that are subjected to oversight by an independent quality assurance unit/person to fulfill the requirements set forth in 21 CFR 58.35. In anticipation for progression to the clinical stage, the investigator is encouraged to (i) use the exact clinical test material or material that closely matches the characteristics of the presumed clinical product, (ii) identify avenues to generate sufficient cGMP product to support an early-stage clinical assessment, and (iii) develop robust assays that are specific, sensitive and reproducible.

\section{Key Sections of an IND Application}

The content and format of an IND submission to the FDA should adhere to 21CFR 312.23 and the application must contain information on three components: (i) Chemistry Manufacturing and Control (CMC), which describes the critical components such as the choice of cells and vector types along with the design and conduct of pre-clinical studies with those components in a manner that is amenable to clinical setting, (ii) pharmacology and toxicological profile of the investigational drug, and (iii) the design of the proposed clinical study such as dosage, administration routes and the manner it will be conducted. Investigators may provide a Letter of Authorization (LoA) for nonclinical sections, which allows the FDA to cross-reference the stated sections of other active INDs or Drug Master Files.

CMC. The contents of this section are to be addressed with respect to the phase of the IND developmental program and the scope of the proposed trial as per 21 CFR 312.23(a) (7)) $[15 \bullet \bullet]$. However, the primary focus at every IND stage is geared towards safety consideration and manufacturing controls associated with the drug. For initial IND submissions, the following key aspects of the investigational drug development are generally expected to be addressed: (i) history, and derivations of starting materials such as cells, 
tissues and viral banks, (ii) detailed description including the mode of action with intended clinical use and characterizations of (i) the drug substance (DS) which is defined as the active pharmacological ingredient, as per 21 CFR 312.23(a) (7)(iv)(a), such as the vector for ex vivo cell manipulation, that gets incorporated into the final drug product, and (ii) drug product (DP), defined as "the finished dosage form" as per 21 CFR 312.23(a)(7)(iv)(b), such as the bioengineered cell product that contains the DS; (iii) manufacturing procedure and controls with respect to phase specific regulatory requirements such as GMP compliance; (iv) analytical tests to assess quality attributes of DS and DP and if needed; (iv) compliance to donor eligibility criteria that depends upon the cell source, whether autologous or allogeneic, as described in 21 CFR 1271 [15••]. It must be noted that the FDA acknowledges that a clear distinction between DS and DP may not be possible for some CGT products and that an explanation of how DS and DP are defined in the IND submission would suffice. The latest guidance document includes FDA's expectations while describing certain aspects of DS and DP such as their manufacturing and analytical test procedures in the IND application [15••].

CGT products are inherently complex and are rife with inherent variables such as (i) homing of the adoptively transferred cells to target sites, (ii) off-target effects, (iii) aberrant proliferation of cells that may lead to tumorigenesis, (iv) interaction with immunodepleting and immunosuppressive agents used prior to adoptive transfer or to counteract overt immune stimulation that may occur after adoptive transfer, respectively, (v) lack of consistent control of ex vivo cell expansion strategies to achieve a clinical starting dose, (vi) inflammatory response to transplanted cells, vii) duration of in vivo persistence of the vector, (viii) potential for vector-borne insertional mutagenesis; and (ix) uneven biodistribution of administered vectors and cells. Therefore, the acquisition of complete CMC data on CGT products are challenging during pre-clinical studies and thereby necessitates an extensive follow up of enrollees in early stages of clinical trials to capture meaningful safety, PK, and efficacy data. Thus, compared to small molecule or antibody-based studies, CGT-based drug development programs are inherently different especially in the context of measuring safety, feasibility, and tolerability [20]. In recognition of the burden on the investigator to gather complete $\mathrm{CMC}$ information to qualify the investigational product for phase 1 or $1 / 2$ trial that employs only small number of participants thereby requiring limited amounts of products while the manufacturing and analytical procedures are still being refined, FDA has waived the requirement to furnish comprehensive CMC data during early-stage IND filing [15••] but does require long-term follow-up of patients to ensure safety.

The manufacturing attributes of an investigational drug is governed by the stage of drug development (Fig. 2). Production of GCT materials are typically performed under, GLP during the pre-clinical development stages and advances to GMP manufacturing procedures for products fit for clinical trials as proposed in the IND application. During such progression in the manufacture of the investigational product, an investigator can encounter changes in product quality that can affect the level of the proposed clinical utility. This finding is not surprising given the changes associated between making stage-specific components in terms of equipment, materials, purification methods, or processes performed differently between the manufacturing grades. The cGMP manufacturing process must adhere to regulatory compliance which is driven by three key aspects.

Raw material practices. FDA defines components as any ingredient used in manufacturing including those that do not appear in the final product. For clinical products, including viral vector products, additional considerations for components are needed. For example, the cell substrate used to manufacture the viral vector must originate from qualified sources (such as Master Cell Bank and Master Working Cell Bank) which has been extensively tested and characterized and shown to be suitable for manufacturing as directed under 21 CFR 210.3(b). To lower or eliminate the risk of zoonotic transmission of adventitious agents, reagents should be free of animal derived components wherever possible and if needed, serum, such as Fetal Bovine Serum, need to be appropriately sourced to avoid the occurrence of bovine spongiform encephalopathy (BSE). These requirements are in addition to normal expectations for control of raw materials (RMs) such as use of appropriate grade (GMP, USP), establishment of RM specifications and unique identifiers of all components used in the batch, qualification of RM
Fig. 2 Manufacturing characteristics of a CGT product from development through market approval

\begin{tabular}{|c|c|c|c|}
\hline $\begin{array}{l}\text { Preclinical } \\
\text { POC }\end{array}$ & $\begin{array}{l}\text { Preclinical } \\
\text { IND } \\
\text { enabling }\end{array}$ & & te \\
\hline$\frac{\text { PI Lab \& Shared }}{\text { Core Facility }}$ & $\frac{\text { Academic GMP }}{\text { Core Facility }}$ & $\frac{\text { Academic \& CDMO }}{\text { GMP Facility }}$ & $\frac{\text { Commercial Entity }}{\text { CDMO Facility }}$ \\
\hline $\begin{array}{l}\text {-Small scale } \\
\text {-Research Grade } \\
\text {-Lab Purified } \\
\text {-Research } \\
\text { Materials }\end{array}$ & $\begin{array}{l}\text {-Modest Scale-Up } \\
\text {-Pilot Grade } \\
\text {-GMP compatible } \\
\text { materials \& } \\
\text { purification }\end{array}$ & $\begin{array}{l}\text {-Phase I/II scale } \\
\text {-Clinical Grade GMP } \\
\text { materials, process, } \\
\text { facility }\end{array}$ & $\begin{array}{l}\text {-Phase III scale } \\
\text {-Commercial Grade and } \\
\text { fully GMP compliant }\end{array}$ \\
\hline
\end{tabular}


suppliers, and the implementation of procedures for the receipt, quarantine, testing, and subsequent release for use or rejection of RM based on testing results. The FDA, in its recognition to the sky-rocketing manufacturing needs of CGT products, has released its latest guidance to reduce the burden of clinical bridging studies when small changes in the manufacturing are made that do not result in transformation of the manufactured product [14]. To safeguard the manufacture of CGT products while the world is being upended by COVID-19 infections, the FDA has published suggestions to prevent product contaminations with the disease causative SARS-CoV-2 virus. Broadly, those recommendations are to (i) review cGMP manufacturing practice to prevent unintended amplification of the viral loads in cellular therapeutic products and (ii) incorporate additional risk assessments to mitigate viral propagation [21]. To date, there is no evidence of such a disease transmission through a pharmaceutical product.

Quality practices. Quality systems (QS) establish confidence in the quality of manufactured products which are predictable and reproducible by operating under pre-determined manufacturing procedures with quality assurance (QA) oversight while the process parameters are continually being evaluated and improved through a controlled and documented process [22]. Such systems can be (i) paper based which is frequently employed in academic core facilities and/or early startups, or (ii) validated software driven operations more commonly employed in larger pharmaceutical manufacturers. Quality systems are implemented to ensure strict control of all GMP sub-systems used in manufacturing and analytical testing. The QS covers various pre-, inprocess, and post-manufacturing operations to ensure quality by design manufacturing and includes, but are not limited to, production and process controls, deviation/corrective action and preventive action (CAPA) management, document control, change control, risk management and facility/equipment controls. As mentioned, modern manufacturing facilities typically integrate and automate their QS using specialized software commonly referred to as a Quality Management System (QMS) that aids with all quality aspects of 21CFR 210 and 211 compliance. These regulations may also apply to suppliers of raw materials procured for cGMP production. The tenants of the QS are instilled by a quality control unit that comprises of QA and quality control (QC) teams. Coordinated efforts between QA and QC impart efficiency in the manufacturing procedures that lead to the production of reliable and safe CGT products. QA is deemed independent and is not subordinate to any groups/units within the organization. Major functions that QA is entrusted to oversee include (i) documentation practices, such as review of batch records (BR), product release certificates and assay reports from qualified quality control laboratories, (ii) review and approval of raw materials used in cGMP manufacturing, and (iii) audits, both internal and external, and analysis of trends to track and uphold the strict performance driven factors essential to produce materials with high quality. The key aspects of QC involve release of raw materials, in-process, and final product release testing and ensure that the manufacture is performed under pre-determined acceptance and specification criteria. Pre-established QC testing procedures fit for the evaluation of traditional pharmaceuticals are difficult to practice with cell and gene therapy products due to their built-in complexities such as limited availability and short life spans of the samples. Therefore, the FDA encourages investigators for mutual engagements to identify and develop novel and/or flexible methods that are conducive for quality determinations of CGT-based IND candidates.

Facilities and Equipment. Manufacturing unit and associated apparatuses must be validated to be compliant with FDA requirements. Such units typically comprise of dedicated areas specific for the type of production such as viral vector production and cell processing. Generally, the logistical and operational requirements for clinical production is beyond the purview of the investigator and is typically assigned to an in-house facility or outsourced to external manufacturers such as contract development and manufacturing organizations (CDMOs) and contract manufacturing organizations (CMOs) that are specialized in this field. However, a general knowledge of the manufacturing process can benefit an investigator to package such information adequately under the $\mathrm{CMC}$ section as required.

Final product testing must occur on materials, such as viral vectors and cellular products, manufactured under cGMP. The primary testing goals are to provide investigational drug-specific information such as (i) safety by providing evidence for sterility, absence of adventitious agents that includes replication competent virus and mycoplasma; (ii) identity by testing for suitable biological features such as cell surface markers; (iii) purity of the composition by detecting for extraneous materials that may have been introduced during the manufacturing process such as endotoxin, protein, growth factors or other factors that may influence final product characteristics; and (iv) potency/product characterization to demonstrate (a) lot-to-lot consistency, (b) comparability if changes are introduced during manufacturing procedures, and (c) clinical suitability for pivotal trials that require drug stability assessments $[15 \bullet \bullet, 23]$. Stability studies, required under 21 CFR 312.23 , are integral to an IND program and are applicable for all IND phases to build quality into products. The goal of such studies is to determine if the CGT product retains pre-established quality limits that are sustained during the proposed clinical trial duration although the intrinsic variabilities in these products 
can pose a challenge to accurately assess their shelf life and storage conditions. These studies are performed on (i) inprocess materials to measure product integrity during the cryopreservation period, and (ii) final product to establish expiration dates. A stability study protocol generally incorporates (i) procedures to (a) capitulate maximum stress conditions and (b) understand degradation via accelerated studies; (ii) testing schedules, that includes evaluation at time zero, and intervening periods followed by a suitable end point; and (iii) justification of assay design, such as the number of lots to test and the suitability of their application. Due to the inherently complex nature of the CGT products, the collection of comprehensive stability data to support the entire period of the proposed human trial may be incomplete or unavailable in some circumstances, such as during preclinical stages. Therefore, the inclusion of a proposed plan to determine stability will fulfill the requirements for initial an IND submission with the provision that the FDA be notified of timely updates on such data as they become available.

As per 21 CFR 312.23(a)(7), the amount of information to be furnished on the IND application is dependent on (i) the phase of the study, (ii) proposed duration and dose, and (iii) available information. For early-phase IND submissions, data reporting with nonvalidated testing methods are allowed provided they incorporate scientifically sound quality attributes, such as specification and acceptance criteria.

Pharmacology and Toxicology (P/T). As set forth in 21CFR 312.23(a)(8), P/T data is a mandatory requirement in an IND application. The primary goal of these studies is to gather safety data of the investigational drug to make informed decision on whether the investigational drug can safely advance to clinical testing [24]. Such data are sourced mainly from appropriate animal models (and cell lines) that are integrated into the pre-clinical studies which must be designed and conducted to support the establishment of a safe starting dose and dose escalation studies with due cognizance of potential toxicities to reduce unwarranted exposure of trial participants to suboptimal dose. Pharmacological evaluation of an investigational drug consists of measurement of its pharmacokinetic (PK) and pharmacodynamic (PD) profile. PK for a traditional pharmaceutic consists of its absorption, distribution, metabolism, and excretion (ADME) profile, whereas PD is defined as the mechanistic pathways by which the drug exerts its action. Due to the inherent variability and novelty in biological features and characteristics of CGT products, it is necessary to customize strategies to determine such pharmacological determinants. Measurements of PK of an investigational CGT product starts with the adoptive transfer of the drug into the appropriate animal model identified during the pre-clinical studies. At suitable time points gathered from the previous studies, the expression, persistence, and tissue biodistribution profile of the vector and the transgene are determined. Any adverse effects that may arise during such study period must be reported and assays are to be developed to relate such events to the viral vector type, transgene expression level and size of the animal cohort. The PD profile is evaluated by measuring the in vivo efficacy of the investigational drug. The aim of toxicity studies is to determine the characteristic (identity and quantity) of the investigational drug in an in vivo setting with respect to potential local and systemic toxicities that may be acute and/or chronic in nature. Evaluation of such studies is helpful to prepare patient monitoring plan.

Clinical Study Design. Design of clinical trials to evaluate CGT investigational products differs from those employed to test traditional pharmaceutical agents. Towards this end, FDA has provided specific regulatory guidance documents to facilitate the preparation of early stage CGT-based IND application [25]. The portfolio of nonclinical studies formulated and performed to identify and capture information, such as $\mathrm{P} / \mathrm{T}$ profile of the investigational drug, critical for its clinical stage advancement, form the basis to develop early phase human trial. Several features of a CGT product such as its characteristics, preclinical considerations, and manufacturing needs drive the design rationale. Such a design takes into account the (i) goals of early-phase trials which are primarily geared to access safety; (ii) evaluation of minimum tolerable dose and dose regimen; (iii) considerations on the characteristics of the clinical trial population such as qualifications and the vetting process to recruit eligible trial participants based on their disease stages and severities, adequacy of response to existing therapies if available, lack of treatment options, physiological and biological considerations amenable to the proposed treatment; (iv) heightened risk associated with a CGT product for a delayed adverse clinical outcome that warrants the formulation of a long term follow-up (LTFU) protocol as exemplified by the FDA mandated time intervals to detect replication competent lentivirus (RCL) in patients administered with retrovirus-based genetically engineered products, and (v) mitigation plan for potential toxicities with established treatment options [26, 27]. Participation of healthy volunteers are discouraged for most CGT-based trials since such therapies are directed to induce permanent or semi-permanent genetic changes while children can only be enrolled if additional safeguards as described in 21 CFR Part 50 are incorporated in the preclinical study designs.

\section{IND Application Stage}

Satisfactory completion the IND-enabling studies leads to the preparation of the dossier to apply for IND designation. To streamline the process of writing and submitting documents required for an IND application, electronic common 
technical document (eCTD) compliant templates that are updated with the current regulatory requirements are commercially available. It is important to recognize that products not intended to be distributed commercially, such as investigator-sponsored INDs, are excluded from the eCTD requirement. Investigators may submit a hardcopy IND submission by mailing their application in triplicate. Alternatively, investigators may pursue DocuSign's Part 11 Module, which is compliant with 21 CFR Part 11 . Host institutions may provide regulatory support, including IND compilation and review, through a Regulatory Affairs Office. Investigators working on investigational drugs that address unmet medical needs are encouraged to seek advantage of special regulatory provisions. FDA has designed four programs to promote and accelerate the development of such drugs to allow their prompt use in patients provided the therapeutic efficacy justify potential risk [28]. The programs that qualify for submission along with the IND application are as follows: (i) "Fast Track" designation which can be based solely upon pre-clinical data. Such a status is bestowed under Sect. 506(b) of the FD\&C Act if the following conditions applicable to the investigational drug are met: (a) it is to treat serious and life-threatening conditions, and (b) it has demonstrated evidence to address unmet medical needs. It is expected that either theoretic/mechanistic rationale, or satisfactory therapeutic outcomes from pre-clinical animal models, or both, would suffice for an academic investigator to request such designation during the early stage IND application. Upon approval of such a request, a window of various interactive opportunities with the FDA opens to the investigator-sponsor to prepare and conduct studies appropriate for such designation, and ii) "Breakthrough Therapy" designation status which is provided under Sect. 506(a) of the FD\&C Act to the investigational drug if it (a) is to treat a serious disorder and (b) has been demonstrated to afford significant improvement over existing drugs in a clinical setting. Therefore, an investigator-sponsor is expected to request for such a designation no earlier than the end of phase 1 but no later than end of phase 2. The other two programs, "Accelerated approval" and "Priority Review" are reserved for FDA's evaluation during product approval stages based on either meeting a well-controlled clinical surrogate end point during late stage human trials or demonstrated findings, submitted with the BLA, that show compared to existing therapies, the investigation product affords significant improvement in critical quality attributes such as safety and efficacy profiles, respectively.

Following the initial submission, any changes to an existing IND application, such as identification of new safety concerns, and manufacturing procedures that can affect product purity and composition, with the potential to influence the core nature of the study proposals must be declared by filling amendments as per $21 \mathrm{CFR} 312.31$. Annual reports are also due, as per 21 CFR 312.33 to summarize any major manufacturing or microbiological changes.

\section{Meeting Opportunities with CBER/OTAT}

The FDA provides and encourages opportunities to discuss various aspects of the study sections, such as re-evaluation of study design, drug safety data, concern for response to drug and any critical/urgent issues for interactions with the stakeholder during early-stage IND development studies as summarized below [29].

\section{Initial Targeted Engagement for Regulatory Advice on CBER Products (INTERACT)}

This is an Informal, early-stage meeting to review features in preliminary studies, CMC, or P/T studies [30].

\section{Pre-IND Meeting}

This is a nonbinding meeting which is typically scheduled at least after the satisfactory completion of preliminary evaluations. The objectives of such a meeting is to obtain input from the FDA on regulatory, and scientific aspects such as advice on the plans for preliminary studies, design of animal studies to support the rationale to advance to clinical testing, and the format for the IND. Questions regarding the clinical protocol can also be discussed.

Meeting Type A. This is a formal meeting to resolve any stalled drug developmental program such as a clinical hold.

Meeting Type B. This is an informal meeting such as in pre-IND stage and formal meeting at the later stage such as at the end of phase 1 (for products with Fast Track designation) and phase 2 .

Meeting Type C. This meeting if for discussion of issues that are beyond the scope of either type A or B such as seeking recommendations to improve upon ongoing issues.

In addition to the established avenues of engagements, it is the FDA's desire to open additional interactive opportunities to improve upon the manufacturing processes and enhance the understanding of critical quality attributes of cell and gene therapy products [31]. Refinements in these categories are critical to bring reproducible clinical outcomes with interventions based on such products. 


\section{Clinical Trials}

The IND dossier is designed to support the rationale and justification to advance the investigational drug for human trials. The CGT product typically must meet trial-phase specific endpoints, which are either clinical or exploratory such as a surrogate marker of the disease, to progress between phases. It is only upon the satisfactory completion of the last testing phase that the drug can qualify for the review of commercial licensure, unless the drug meets specifications for expedited approval mechanisms that exist in the early stages. Below is a brief discussion of clinical phases that are within the scope of early stage drug development by an investigator-sponsor.

Phase 1. The primary goal at this stage is to evaluate the safety and tolerance of the CGT product in recruited trial participants. The drug product is administered at the proposed starting dose and may include dosing escalations. The best practice in the selection of a dosing strategy is built upon the product characteristics and the intervention strategy such as the safety profile of the IND product and its route of administration. A cohort of very few (around 10 or less) to 80 participants, selected based on factors such as disease prevalence, funding, and existing clinical data, are enrolled and their pharmacological and toxicological evaluations are closely monitored for unwarranted adverse outcomes. Besides drug safety assessments, a secondary objective is the preliminary monitoring of indications for drug efficacy and such a data can influence the design of later stages of a clinical trial. Due to the uniqueness in CGT products such as their persistence, biodistribution and durability of action, the probability of success in early clinical phases often times relies on the outcomes from well-defined pre-clinical studies such as the derivation of a starting dose with minimal risk from the organ toxicity profiles of pre-clinical animal models. Amount of CGT products required to conduct this phase are within the production capacities of academic CGT manufacturing units. In addition, phase 1 testing should identify drug dosing to be used in phase 2 testing.

Phase 2. The primary goals of this trial is to continue (i) phase 1 safety assessments and evaluation of the effectiveness of the CGT product on a larger cohort size that can range up to a few hundreds. To accommodate the scale of materials required for this phase such as viral vectors and cell products, third-party manufacturers rather than academic core facilities, may be more suitable. The investigatorsponsor should be aware of escalation in operational requirements and pre-plan for budgetary and logistical resources in order to successfully navigate such a phase. (ii) In addition, phase 2 studies should identify the drug dose to be used in pivotal phase 3 testing.

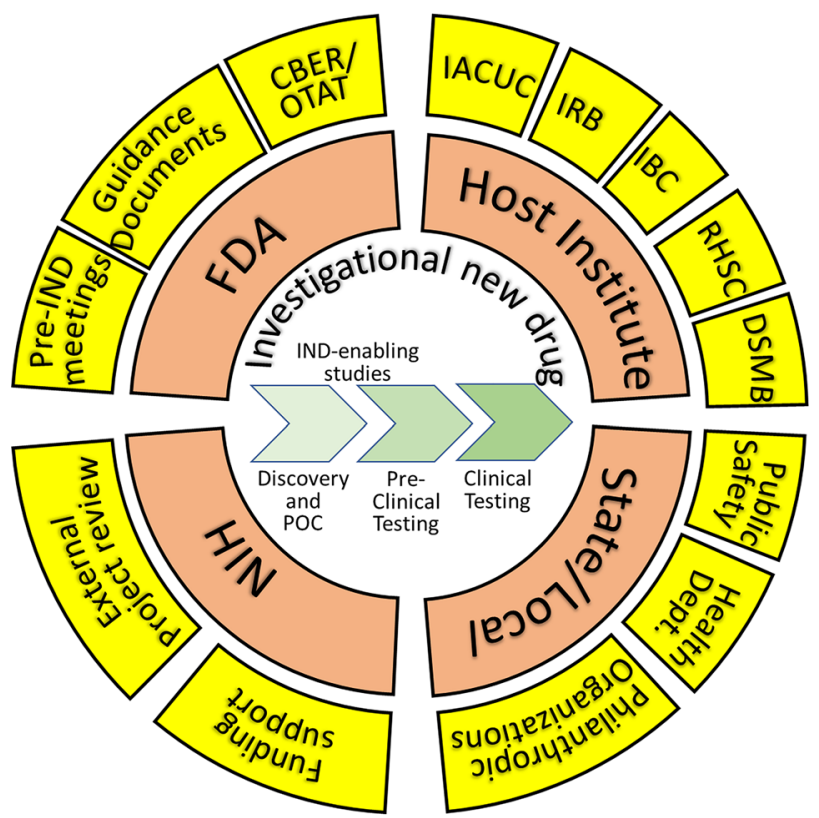

Fig. 3 Representation of the processes and agencies working together for the successful clinical testing of a drug product candidate

Phase 3. The main goals at this stage are to continue to collect data on the safety and efficacy profile of the investigational drug. The scope and magnitude of the trial parameters at this phase are wide such that it (i) is designed to be tested in various formats such as controlled, uncontrolled, and expanded; (ii) recruits hundreds to thousands of participants; and (iii) is conducted across multiple centers and sites. Activities necessary to conduct phase 3 studies are beyond the scope of this article as are phase 4 post-licensure studies after market approval and requires extensive operational resources that are usually managed outside of the host institution of the investigator-sponsor.

\section{Conclusion}

CGT-based therapeutics are transformative medicines with life-altering prospects. Innovators engaged in bringing such disruptive medical technologies must have a wellchartered path to navigate their investigational products for FIH assessment via the IND mechanism (which is summarized in Fig. 3). Due to the uniqueness of CGT products, a well-planned IND program must address the technical and logistical hurdles that are intrinsic to their developmental processes. Advancements in vector design and selection, delivery mechanisms of CGT products, and their manufacturing strategies are potential avenues to increase efficiency in evaluating such innovative products. An investigator well versed in scientific skills must also be proficient on the 
knowledge of regulatory oversight mechanisms set forth by the FDA, which is committed to safeguarding the health of the nation while promoting exploration of innovative therapies. Towards this end, fostering a robust investigator-FDA partnership early during the preliminary evaluation stages of drug development is a mission critical to fulfill the tenets set forth in the IND application.

\section{Declarations}

Conflict of Interest Dr. Doering reports other from Expression Therapeutics, Inc., during the conduct of the study. The other authors declare that they have no conflict of interest.

Human and Animal Rights and Informed Consent This article does not contain any studies with human or animal subjects performed by any of the authors.

\section{References}

\section{Papers of particular interest, published recently, have been highlighted as: \\ - Of importance \\ $\bullet$ Of major importance}

1. Bailey AM, Arcidiacono J, Benton KA, Taraporewala Z, Steve WS. United States Food and Drug Administration regulation of gene and cell therapies. Adv Exp Med Biol. 2015;871:1-29.

2. Verma IM. A Tumultuous year for gene therapy. Mol Ther. 2000;2(5):415-6.

3. Marwick CBMJ. FDA halts gene therapy trials after leukemia case in France. 2003;326(7382):181.

4. Cole A. Child in gene therapy programme develops leukaemia. BMJ. 2008;336(7634):13.

5. Dunbar CE, High KA, Joung JK, Kohn DB, Ozawa K, Sadelain M. Gene therapy comes of age. Science. 2018;359(6372):eaan4672.

6. News in Brief. High-dose AAV gene therapy deaths. Nat Biotechnol. 2020;38:910.

7. Philippidis A. "Profoundly saddened" Lysogene discloses child's death in phase II/III trial. Hum Gene Ther. 2020;31(21-22):1141-3.

8. Galaro AK, Christopher SC. FDA perspective on the preclinical development of cell-based immunotherapies. Cell \& Gene Therapy Insights. 2020;6(9):1381-90. https://doi.org/10.18609/ cgti.2020.148.

9. Lapteva L, Purohit-Sheth T, Serabian M, Puri RK. Clinical development of gene therapies: the first three decades and counting. Mol Ther Methods Clin Dev. 2020;10(19):387-97.

10. S. Gottlieb. Statement from FDA Commissioner Scott Gottlieb, MD and Peter Marks, MD, PhD, Director of the Center for Biologics Evaluation and Research on New Policies to Advance Development of Safe and Effective Cell and Gene Therapies. 2019. https://www.fda.gov/news-events/press-announcements/ statement-fda-commissioner-scott-gottlieb-md-and-peter-marksmd-phd-director-center-biologics. Accessed 7 Feb 2021. Given the surge in the exploration of cell and gene therapy-based modalities FDA has issued this forward-looking statement on its mission to modernize the regulatory framework and to reiterate its commitment to assist the stakeholders as they develop their INDs.
11. U.S. Food and Drug Administration. Approved cellular and gene therapy products. 2021. https://www.fda.gov/vaccines-blood-biologics/ cellular-gene-therapy-products/approved-cellular-and-gene-therapyproducts. Accessed 7 Feb 2021.

12. Holbein BME. Understanding FDA regulatory requirements for investigational new drug applications for sponsor-investigators. J Investig Med. 2009;57(6):688-94.

13. U.S. Food and Drug Administration, Guidance. https://www.fda. gov/industry/fda-basics-industry/guidances. Accessed 7 Feb 2021.

14. U.S. Food and Drug Administration. FDA continues strong support of innovation in development of gene therapy products. 2020. https:// www.fda.gov/news-events/press-announcements/fda-continues-strongsupport-innovation-development-gene-therapy-products. Accessed 7 Feb 2021.

15.••U.S. Department of Health and Human Services Food and Drug Administration Center for Biologics Evaluation and Research. Chemistry, Manufacturing, and Control (CMC) Information for Human Gene Therapy Investigational New Drug Applications (INDs), Guidance for Industry. 2020. https://www.fda.gov/media/ 113760/download. Accessed 7 Febr 2021. With the overarching goal being to streamline the review and approval process to enhance promising treatment entry into the clinic, this is FDA's latest guidance for recommendations on the CMC section of a gene therapy IND application.

16. Huang W, Percie du Sert N, Vollert J, Rice ASC. General principles of preclinical study design. Handb Exp Pharmacol. 2020;25755-69.

17. U.S. Department of Health and Human Services Food and Drug Administration Center for Biologics Evaluation and Research. Preclinical assessment of investigational cellular and gene therapy products, Guidance for Industry. 2013. https://www. fda.gov/media/87564/download. Accessed 7 Feb 2021.

18. U.S. Department of Health and Human Services Food and Drug administration, Office of Regulatory affairs. Good laboratory practices questions and answers, Guidance for Industry 1981; June (Minor editorial and formatting changes made December1999 \& 2007; July). https://www.fda.gov/media/75866/download. Accessed 7 Feb 2021.

19. United Sates Department of Health and Human Services, Food and Drug Administration. Good laboratory practice for nonclinical laboratory studies; Proposed Rule. 2016. https://www.fda.gov/ media/99828/download. Accessed 7 Feb 2021.

20. Husain SR, J Han J, Au P, Shannon K, Puri RK. Gene therapy for cancer: regulatory considerations for approval. Cancer Gene Therapy. 2015;22:554-63.

21. U.S. Department of Health and Human Services, Food and Drug Administration, Center for Biologics Evaluation and Research. Manufacturing considerations for licensed and investigational cellular and gene therapy products during COVID-19 public health emergency, Guidance for Industry. 2021. https://www.fda.gov/ media/145301/download. Accessed 7 Feb 2021.

22. U.S. Department of Health and Human Services, Food and Drug Administration, Center for Drug Evaluation and Research (CDER), Center for Biologics Evaluation and Research (CBER), Center for Veterinary Medicine (CVM). Quality systems approach to pharmaceutical current good manufacturing practice regulations, Guidance for Industry. 2006. https://www.fda.gov/media/ 71023/download. Accessed 7 Feb 2021.

23. U.S. Department of Health and Human Services Food and Drug Administration Center for Biologics Evaluation and Research. Potency tests for cellular and gene therapy products, guidance for industry. 2011. https://www.fda.gov/media/79856/download .Accessed $7 \mathrm{Feb} 2021$.

24. National gene vector Biorepository. Approach to preclinical evaluation for gene therapy (GT) products, FDA Regulations and Guidance's. https://www.ngvbcc.org/pdf/NGVB_Pharm_Tox.PreClin doc2.17.11.pdf. Accessed 7 Feb 2021. 
25. U.S. Department of Health and Human Services, Food and Drug Administration, Center for Biologics Evaluation and Research. Guidance for industry: considerations for the design of early-phase clinical trials of cellular and gene therapy products, Guidance for Industry. 2015. https://www.fda.gov/media/106369/download. Accessed 7 Feb 2021.

26. U.S. Department of Health and Human Services Food and Drug Administration Center for Biologics Evaluation and Research. Long term follow-up after administration of human gene therapy products, guidance for industry. 2020. https://www.fda.gov/media/ 113768/download. Accessed 7 Feb 2021.

27. U.S. Department of Health and Human Services Food and Drug Administration Center for Biologics Evaluation and Research. Testing of retroviral vector-based human gene therapy products for replication competent retrovirus during product manufacture and patient follow-up, Guidance for Industry. 2020. https://www. fda.gov/media/113790/download. Accessed 7 Feb 2021.

28. U.S. Department of Health and Human Services Food and Drug Administration Center for Drug Evaluation and Research (CDER) Center for Biologics Evaluation and Research (CBER). Expedited programs for serious conditions - drugs and biologics, Guidance for Industry. 2014. https://www.fda.gov/media/86377/download. Accessed 7 Feb 2021.
29. U.S. Department of Health and Human Services, Food and Drug Administration, Center for Drug Evaluation and Research (CDER), Center for Biologics Evaluation and Research (CBER). IND Meetings for Human Drugs and Biologics, Chemistry, Manufacturing, and Controls Information, Guidance for Industry. 2001. https://www.fda.gov/media/70827/download. Accessed 7 Feb 2021.

30. U.S. Food and Drug Administration, Center for Biologics Evaluation and Research. SOPP 8214: INTERACT Meetings with Sponsors for Drugs and Biological Products. 2020. https://www.fda. gov/media/124044/download. Accessed 7 Feb 2021.

31. Marks Peter. A year like no other: reflecting on the challenges of 2020, and looking to regain momentum for cell and gene therapy in 2021. Cell and Gene Therapy Insights. 2021. https:// www.insights.bio/cell-and-gene-therapy-insights/journal/article/ 1892/A-year-like-no-other-reflecting-on-the-challenges-of-2020and-looking-to-regain-momentum-for-cell-and-gene-therapy-in2021. Accessed 7 Feb 2021.

Publisher's Note Springer Nature remains neutral with regard to jurisdictional claims in published maps and institutional affiliations. 